\title{
Pythagorean Orthogonality in a Normed Linear. Space
}

\author{
By Hazel Perfect
}

(Received 5th September, 1956.)

This note presents a proof of the following proposition:

THeorem. If Pythagorean orthogonality is homogeneous in a normed linear space $T$ then $T$ is an abstract Euclidean space.

The theorem was originally stated and proved by R. C. James ([1], Theorem 5.2) who systematically discusses various characterisations of a Euclidean space in terms of concepts of orthogonality. I came across the result independently and the proof which I constructed is a simplified version of that of James. The hypothesis of the theorem may be stated in the form:

$$
\begin{aligned}
& \text { If }\|x\|^{2}+\|y\|^{2}=\|x-y\|^{2} \text {, then for all complex numbers } \lambda, \mu \\
& \|\lambda x\|^{2}+\|\mu y\|^{2}=\|\lambda x-\mu y\|^{2} \text {. }
\end{aligned}
$$

Since a normed linear space is known to be Euclidean if the parallelogram law:

$$
\|x-y\|^{2}+\|x+y\|^{2}=2\left(\|x\|^{2}+\|y\|^{2}\right)
$$

is valid throughout the space (see [2]), it is evidently sufficient to show that (1) implies (2).

Proof. Let $x, y \varepsilon T$; assume that $\|x\| \geqslant\|y\|$, and consider the (continuous) function

$$
f(\lambda)=\|x-(y+\lambda x)\|^{2}-\|x\|^{2}-\|y+\lambda x\|^{2}
$$

of the real variable $\lambda$.

(i) Suppose $f(0)=\|x-y\|^{2}-\|x\|^{2}-\|y\|^{2} \geqslant 0$.

Then since $f(1)=\|y\|^{2}-\|x\|^{2}-\|x+y\|^{2} \leqslant 0$, it follows that there exists $\lambda$ in the interval $[0,1]$ such that $f(\lambda)=0$.

(ii) Suppose $f(0)<0$. 
Pythagorean Orthogonality in a Normed Lingar Space 169

If $f(-1) \geqslant 0$ then there exists $\lambda$ in the interval $[-1,0)$ such that $f(\lambda)=0$.

On the other hand if $f(-1)=\|2 x-y\|^{2}-\|x\|^{2}-\|x-y\|^{2}<0$ then

$$
\begin{aligned}
f(-2) & =\|3 x-y\|^{2}-\|x\|^{2}-\|2 x-y\|^{2} \\
& >\|3 x-y\|^{2}-2\|x\|^{2}-\|x-y\|^{2} \\
& >\|3 x-y\|^{2}-3\|x\|^{2}-\|y\|^{2} \\
& \geqslant\|3 x\|^{2}+\|y\|^{2}-2\|3 x\| \cdot\|y\|-3\|x\|^{2}-\|y\|^{2} \\
& =6\|x\|(\|x\|-\|y\|) \geqslant 0 ;
\end{aligned}
$$

and so there exists $\lambda$ in the interval $[-2,-1)$ such that $f(\lambda)=0$.

Now let $\lambda^{*}$ be any real zero of the function $f(\lambda)$ and write $z=y+\lambda^{*} x$, so that $\|x\|^{2}+\|z\|^{2}=\|x-z\|^{2}$.

Then

$$
\begin{aligned}
\|x-y\|^{2}+\|x+y\|^{2} & =\left\|x-z+\lambda^{*} x\right\|^{2}+\left\|x+z-\lambda^{*} x\right\|^{2} \\
& =\left\|x\left(1+\lambda^{*}\right)-z\right\|^{2}+\left\|x\left(1-\lambda^{*}\right)+z\right\|^{2} \\
\text { [by (1)] } \quad & =\left(1+\lambda^{*}\right)^{2}\|x\|^{2}+\|z\|^{2}+\left(1-\lambda^{*}\right)^{2}\|x\|^{2}+\|z\|^{2} \\
& =2\|x\|^{2}+2\left(\lambda^{* 2}\|x\|^{2}+\|z\|^{2}\right) \\
\text { [by (1)] } & =2\|x\|^{2}+2\|y\|^{2} ;
\end{aligned}
$$

[by (1)]

which establishes (2) and thus completes the proof of the theorem.

\section{REFERENCES.}

[1] R. C. James, "Orthogonality in normed linear spaces ", Duke Math. J., 12 (1945), 291-302.

[2] P. Jordan and J. von Neumann, "On inner products in linear metric spaces ", Annals of Math., 36 (1935), 719-723.

Untversity College,

Swansea. 